\title{
SINGULAR MEASURES WITH SPECTRAL GAPS
}

\author{
RUSSELL LYONS
}

(Communicated by Richard R. Goldberg)

\begin{abstract}
We show that every Borel measure on the circle whose Fourier spectrum has lacunary-type gaps annihilates every $H$-set.
\end{abstract}

In the study of sets of uniqueness on the circle $\mathbf{T}=\mathbf{R} / \mathbf{Z}$, Rajchman's class of examples, the $H$-sets, continues to play a predominant role. Recall their definition [1, Chapter IX]:

DEfinition. A Borel set $E \subset \mathbf{T}$ is said to be an $H$-set if there exist a strictly increasing sequence of integers $\left\{m_{j}\right\}$ and a nonempty open arc $I \subset \mathbf{T}$ such that for all $x \in E, m_{j} x \notin I$.

Thus, Cantor's middle-thirds set arises from the choices $m_{j}=3^{j}$ and $\left.I=\right] \frac{1}{3}, \frac{2}{3}[$. To say that $H$-sets are sets of uniqueness is to say that they do not support any pseudofunction (i.e., any distribution whose Fourier coefficients vanish at infinity) [1, Chapter IV]. Here we shall show further that $H$-sets do not support (and indeed are annihilated by) any measure whose spectrum has certain lacunary-type gaps. In fact, strict gaps will not be necessary, but merely the condition (1) below. Let $M(\mathbf{T})$ denote the set of complex Borel measures on $\mathbf{T}$.

THEOREM 1. Let $\mu \in M(\mathbf{T})$. If there is a set $A \subset \mathbf{Z}^{+}$such that

$$
\lim _{\substack{n \rightarrow \infty \\ n \in \mathbf{Z}^{+} \backslash A}} \hat{\mu}(n)=0
$$

and such that $A$ can be written as

$$
A=\bigcup_{k \geq 1}\left[c_{k}, d_{k}\right]
$$

with

$$
\inf _{k} \frac{c_{k+1}}{d_{k}}>1
$$

and

$$
\sup _{k} \frac{d_{k}}{c_{k}}<\infty
$$

then $\mu E=0$ for all $H$-sets $E$.

An example of such a measure is given by a Riesz product [2, p. 107]

$$
\mu=\prod_{k \geq 1}\left(1+\operatorname{Re}\left\{\alpha_{k} e^{2 \pi i n_{k} t}\right\}\right)
$$

Received by the editors August 25, 1987.

1980 Mathematics Subject Classification (1985 Revision). Primary 43A46; Secondary 42 A55. Research partially supported by an American Mathematical Society Research Fellowship. 
with $\inf n_{k+1} / n_{k}>3$ and $\left|\alpha_{k}\right| \leq 1$, thereby extending [4, Theorem 1 and $\mathbf{5}$, Theorem 9].

We shall require a theorem of Levinson on the relation of the support of a measure to its spectrum. In order to state it, identify $\mathbf{T}$ with $[0,1[$ and extend the Fourier transform to $\mathbf{R}$ by

$$
\hat{\mu}(\xi)=\int_{0}^{1} e^{-2 \pi i \xi x} d \mu(x)
$$

Given a sequence $\left\{\xi_{k}\right\} \subset \mathbf{R}^{+}$, we define its maximum density $D$ by

$$
D=\lim _{\theta \rightarrow 1^{-}} \limsup _{u \rightarrow \infty} \frac{\operatorname{card}\left\{k: \theta u<\xi_{k} \leq u\right\}}{u-\theta u}
$$

and its minimum density $d$ by

$$
d=\lim _{\theta \rightarrow 1^{-}} \liminf _{u \rightarrow \infty} \frac{\operatorname{card}\left\{k: \theta u<\xi_{k} \leq u\right\}}{u-\theta u} .
$$

THEOREM 2 [3, pp. 13-14]. Let $\mu \in M(\mathbf{T})$ and let $D$ be the maximum density of $\{\xi>0: \hat{\mu}(\xi)=0\}$. Then $\mu$ is not supported by any arc of length less than $D$. In particular, if $D=1$, then $\operatorname{supp} \mu=\mathbf{T}$.

(For definitive results in this direction due to Beurling and Malliavin, see [6]. In the literature, Theorem 2 is stated only for functions. However, the case of general measures follows easily from this. Alternatively, the same proof works for general measures.)

Levinson's theorem resembles the uncertainty principle in that the smaller $\{\hat{\mu} \neq$ $0\}$ is, the more $\{\mu \neq 0\}$ must "spread out." Theorem 1 also follows this heuristic, but is particular to singular measures. (A similar theorem that "arithmetic" gaps of a certain type in the spectrum of a measure force all Helson sets to have measure zero appears in [4].)

Theorem 1 is an instance of a more general theorem, for which we use the following notation: $\forall^{e} j$ means "for all but a finite number of $j$."

THEOREM 3. Let $\mu \in M(\mathbf{T})$. If there is a set $A \subset \mathbf{Z}^{+}$such that (1) holds and such that for any strictly increasing sequence $\left\{m_{j}\right\} \subset \mathbf{Z}^{+}$and any choices of $b_{r} \in \mathbf{Z}$ $(r \geq 1)$, the minimum density of

$$
\mathscr{S}=\left\{r \in \mathbf{Z}^{+}: \forall^{e} j r m_{j}+b_{r} \in A\right\}
$$

is zero, then $\mu E=0$ for all $H$-sets $E$.

PROOF. In [5, Theorem 13], we gave the following necessary and sufficient condition that a measure $\mu$ annihilate all $H$-sets: whenever $\sigma_{x} \in M(\mathbf{T})$ for $\mu$-a.e. $x$ and $m_{j} \rightarrow \infty$ are such that

$$
\forall r \in \mathbf{Z} e^{-2 \pi i r m_{j} x} \rightarrow \hat{\sigma}_{x}(r) \text { weak }^{*} \text { in } L^{\infty}(\mu),
$$

then $\operatorname{supp} \sigma_{x}=\mathbf{T} \mu$-a.e. Thus, suppose that $A$ satisfies the hypotheses of the theorem, that $m_{j} \rightarrow \infty$, and that (5) holds. Suppose that $r \in \mathbf{Z}^{+}$is such that $\hat{\sigma}_{x}(r) \neq 0$ for $\mu$-many $x$. Then for some $b_{r}$,

$$
0 \neq \int_{\mathbf{T}} \hat{\sigma}_{x}(r) e^{-2 \pi i b_{r} x} d \mu(x)=\lim _{j \rightarrow \infty} \hat{\mu}\left(r m_{j}+b_{r}\right),
$$


whence $\forall^{e} j r m_{j}+b_{r} \in A$ by (1). Making these choices for $b_{r}$ when $\hat{\sigma}_{x}(r) \not \equiv 0$ and taking $b_{r}=0$ otherwise, we see that the intersection of the spectrum of $\sigma_{x}$ with $\mathbf{Z}^{+}$ is contained in $\mathscr{S}$ for $\mu$-a.e. $x$. Since $\mathbf{Z}^{+} \backslash \mathscr{S}$ has maximum density one, Theorem 2 shows that $\operatorname{supp} \sigma_{x}=\mathbf{T} \mu$-a.e. This completes the proof.

PROOF OF THEOREM 1. Let $A$ satisfy the hypotheses. In order to apply Theorem 3, let $m_{j} \rightarrow \infty$, let $b_{r} \in \mathbf{Z}$, and let $\mathscr{S}$ be as in (4). Let the elements of $\mathscr{S}$ be $\left\{r_{l}\right\}_{l \geq 1}$ in increasing order. Our task is to show that $\mathscr{S}$ has minimum density zero, which we shall do by showing that $\lim _{\sup _{l \rightarrow \infty}} r_{l+1} / r_{l}>1$.

Let $q=\inf c_{k+1} / d_{k}$ and $Q=\sup d_{k} / c_{k}$. Then if $\limsup _{l \rightarrow \infty} r_{l+1} / r_{l}=1$, we would have that for some $L, r_{l+1} / r_{l}<q$ for all $l \geq L$. Choose $p$ so large that $r_{L+p} / r_{L}>Q$. By definition of $A$ and because $m_{j} \rightarrow \infty$, we may take $j$ so large that

$$
\begin{gathered}
\text { for } L \leq l \leq L+p, \quad r_{l} m_{j}+b_{r_{l}} \in A \\
\text { for } L \leq l \leq L+p, \quad 1<\frac{r_{l+1} m_{j}+b_{r_{l+1}}}{r_{l} m_{j}+b_{r_{l}}}<q
\end{gathered}
$$

and

$$
\frac{r_{L+p} m_{j}+b_{r_{L+p}}}{r_{L} m_{j}+b_{r_{L}}}>Q .
$$

Then for each $l \in[L, L+p]$, it follows from (6) and (7) that there is some $k$ such that both $r_{l} m_{j}+b_{r_{l}}, r_{l+1} m_{j}+b_{r_{l+1}} \in\left[c_{k}, d_{k}\right]$. Hence all $r_{l} m_{j}+b_{r_{l}}$ belong to the same $\left[c_{k}, d_{k}\right]$, whence

$$
\frac{r_{L+p} m_{j}+b_{r_{L+p}}}{r_{L} m_{j}+b_{r_{L}}} \leq \frac{d_{k}}{c_{k}} \leq Q .
$$

This contradicts (8), and so $\limsup _{l \rightarrow \infty} r_{l+1} / r_{l}>1$.

\section{REFERENCES}

1. C. C. Graham and O. C. McGehee, Essays in commutative harmonic analysis, Springer-Verlag, Berlin and New York, 1979.

2. Y. Katznelson, An introduction to harmonic analysis, 2nd corrected ed., Dover, New York, 1976.

3. N. Levinson, Gap and density theorems, Amer. Math. Soc. Colloq. Publ., vol. 26, Amer. Math. Soc., Providence, R. I., 1940.

4. R. Lyons, The size of some classes of thin sets, Studia Math. 86 (1987), 59-78.

5. __ Mixing and asymptotic distribution modulo 1, Ergodic Theory Dynamical Systems (to appear).

6. R. M. Redheffer, Completeness of sets of complex exponentials, Adv. in Math. 24 (1977), 1-62.

7. A. Zygmund, Trigonometric series, 2nd ed., reprinted, Volumes I, II, Cambridge Univ. Press, Cambridge, 1979.

Department of Mathematics, Stanford University, Stanford, California 